\title{
New Numerical Methods for Quantum Field Theories on the Continuum*
}

\author{
P. Emirdağ ${ }^{\mathrm{a}}$, R. Easther ${ }^{\mathrm{a}}$, G. S. Guralnik ${ }^{\mathrm{a}}$, S. C. Hahn ${ }^{\mathrm{a}}$ \\ aDepartment of Physics, Brown University, Providence, RI, USA 02912-1843
}

The Source Galerkin Method is a new numerical technique that is being developed to solve Quantum Field Theories on the continuum. It is not based on Monte Carlo techniques and has a measure to evaluate relative errors. It promises to increase the accuracy and speed of calculations, and takes full advantage of symmetries of the theory. The application of this method to the non-linear $\sigma$ model is outlined.

\section{MOTIVATION}

Conventionally, in numerical approaches to field theories, integrals for generating functionals are evaluated on lattices using Monte Carlo methods. These are quite successful but there are still constraints due to lack of computer power and algorithmic limitations. These limitations become important for theories with significant contributions from fermionic interactions beyond the quenched approximation, and for actions that are not positive definite. Source Galerkin is an alternative computational method that avoids these particular problems [1,2]. It works both on the lattice and the continuum and handles fermions as easily as bosons [3:4. Computations require significantly less time than Monte Carlo based methods. The basic idea of the method is to find solutions to the functional differential equations for a field theory in the presence of sources. The solutions are generating functionals of the theory.

\subsection{Overview of the Method}

To analyze a theory, we start with a Lagrangian in the presence of external sources. The source functional differential equations satisfied by $Z$ are generated in the usual way. The Source Galerkin technique consists of a systematic iterative way to solve these equations to increasing accuracy. We proceed by introducing an ansatz which is an

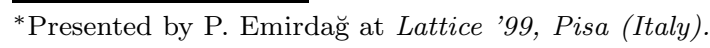

expansion over the external sources of the theory,

$$
Z^{*}=\exp \left[\int j_{x} G_{x y} j_{y}+\int j_{\omega} j_{x} H_{\omega x y z} j_{y} j_{z}+\cdots\right]
$$

$Z^{*}$ does not satisfy the source differential equations of the field theory exactly, but we can require that specific functional projections of these equations are satisfied. These projections are formed by multiplying by test functions in sources and integrating this product in source space using a fixed measure. Introducing the appropriate number of test functions, we can solve for the parameters of the resulting expressions. This method causes the error in assumed solutions to approach zero in the mean and so the ansätz satisfies the equations approximately. The details of this will be made clear in what follows. This approach belongs to the general class of techniques called Galerkin methods [5].

\section{APPLICATIONS}

The method has been applied to various field theories on the lattice and, more recently, on the continuum $\lambda \phi^{4}$, Gross-Neveu, and other two dimensional fermionic models have been studied. Here, we discuss an application of the method to the $O(3)$ Nonlinear $\sigma$ Model with $d=2$. Since this model is asymptotically free, it serves as a toy model for our initial approach to Non-Abelian gauge theories. 


\section{1. $O(3)$ Nonlinear $\sigma$ Model $(d=2)$}

This model consists of three real fields coupled to each other. The Lagrangian is:

$$
\begin{aligned}
L= & \frac{1}{2} \partial_{\mu} \phi^{a}(x) \partial^{\mu} \phi^{a}(x)+j^{a}(x) \phi^{a}(x) \\
& +\frac{1}{2} \chi(x)\left(\phi^{a}(x) \phi^{a}(x)-\frac{1}{g^{2}}\right)-\chi(x) S(x)
\end{aligned}
$$

$\phi$ and $\chi$ are the canonical and Lagrange multiplier fields respectively. $j$ and $S$ are the external sources for these fields while $g$ is the coupling constant of the theory. The Schwinger-Dyson equations are

$$
\begin{gathered}
\partial^{2} \frac{\delta Z[j, S]}{\delta j^{a}(x)}-\frac{\delta^{2} Z[j, S]}{\delta j^{a}(x) \delta S(x)}-j^{a}(x) Z[j, S]=0, \\
\frac{\delta^{2} Z[j, S]}{\delta j^{a}(x) \delta j^{a}(x)}-\frac{1}{g^{2}} Z[j, S]-2 S(x) Z[j, S]=0 .
\end{gathered}
$$

These are the functional differential equations to which we apply the Galerkin procedure. The simplest ansätz is

$$
Z=\exp \left[\frac{1}{2} \int j^{a}(x) G^{a b}(x, y) j^{b}(y)+\chi_{0} \int S(x)\right] .
$$

The inner product that will be used for projecting residuals on the test functions is

$$
(A[j], B[j])_{j}=\int[d j] e^{-j^{2} / \epsilon^{2}} A[j] B[j] .
$$

The subscript denotes the $j$ space. $\epsilon$ weights the inner product such that higher order terms contribute less. For this model, the projections are

$$
\begin{gathered}
\left(1,\left(j^{b}\left(x^{\prime}\right), R_{a}\right)_{j}\right)_{S}=0, \\
\left(1,\left(1, R_{c}\right)_{j}\right)_{S}=0 .
\end{gathered}
$$

$R_{a}$ and $R_{c}$ are the residuals for the equations of motion and constraint respectively. These equations, are solved for Green's functions and Lagrange multiplier, $\chi_{0}$, and are defined in source and coordinate space. The space-time dependence of the Green's functions is approximated by truncating a Sinc expansion,

$$
G(x)=\sum_{k=-N}^{N} G(k h) S(k, h)(x) .
$$

where the Sinc function is defined as

$$
S(k, h)(x)=\frac{\sin \pi(x-k h) / h}{\pi(x-k h) / h} .
$$

Sinc functions have many algebraic properties that make them very useful to work with [6]. While this is a very direct approach, this interpolative approximation requires that every extra dimension introduces another sum to the problem. Using this approximation $\mathcal{O}\left(N^{d}\right)$ numbers are required to store a two point function. The memory limitations become a concern when $\mathcal{O}\left(N^{6 d}\right)$ numbers are to be stored for the Jacobian with four-point functions. This leads us to seek an alternative method which uses general information about the spectral structure of field theories. Any exact two point function can be represented as a sum over free two point functions using spectral representations. Consequently, we can shift our basis for numerical solutions to regulated free Euclidean Green's functions:

$$
G(x)=\frac{1}{(2 \pi)^{d}} \int d p \frac{e^{-p^{2} / \Lambda^{2}+i p x}}{p^{2}+m^{2}}
$$

A term in the spectral representations has the Sinc expansion:

$$
\begin{aligned}
& G(x) \approx G_{0} \sum_{k=-N}^{N} c(k) \exp \left[-\frac{x^{2}}{4\left(e^{k h}+1 / \Lambda^{2}\right)}\right] \\
& c(k)=\frac{1}{e^{k h}}\left[\frac{\pi}{e^{k h}+1 / \Lambda^{2}}\right]^{d / 2} \exp \left[-e^{k h} m^{*^{2}}\right]
\end{aligned}
$$

The Galerkin equations are solved approximately using only one term of the spectral expansion with the parameters $m^{*^{2}}$ and $G_{0}$. The $\beta$ function (Figure. 1) is calculated and compared to $1 / N$ results. The ansätz is improved,

$$
\begin{aligned}
Z^{*} & =Z_{s}^{*} \exp \left[\frac{1}{2} \int S(x) D(x-y) S(y)\right. \\
& \left.+\frac{1}{2} \int j^{a}(x) j^{b}(y) F^{a b}(x-z, y-z) S(z)\right] .
\end{aligned}
$$

$Z_{s}$ is the simplest ansätz. $F^{a b}$ and $D$ are the vertex term and the two-point function for the $\chi$ field respectively. Results get close to exact value dramatically with improved ansätz. We have shown this in the $\lambda \phi^{4}$ model by introducing a four-point function (Figure. 2). 


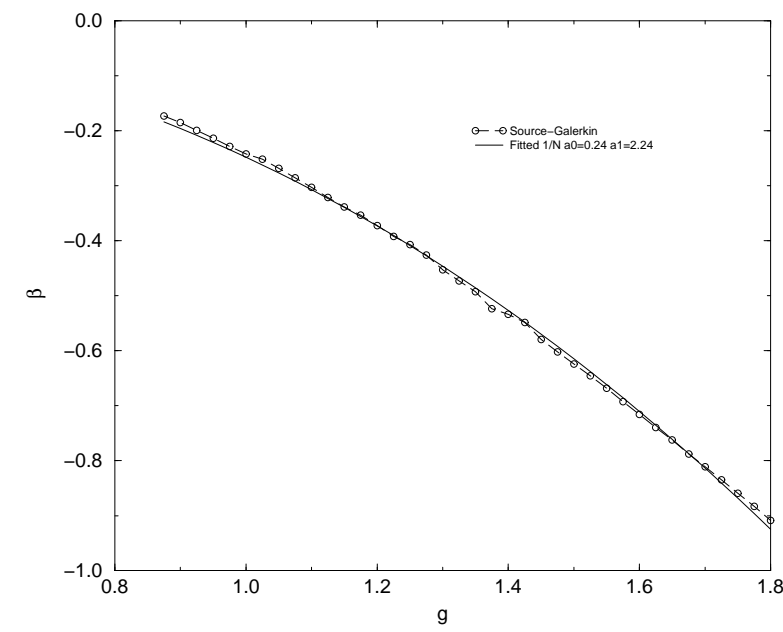

Figure 1. $\beta$ function vs. coupling, compared to $1 / N$ result.

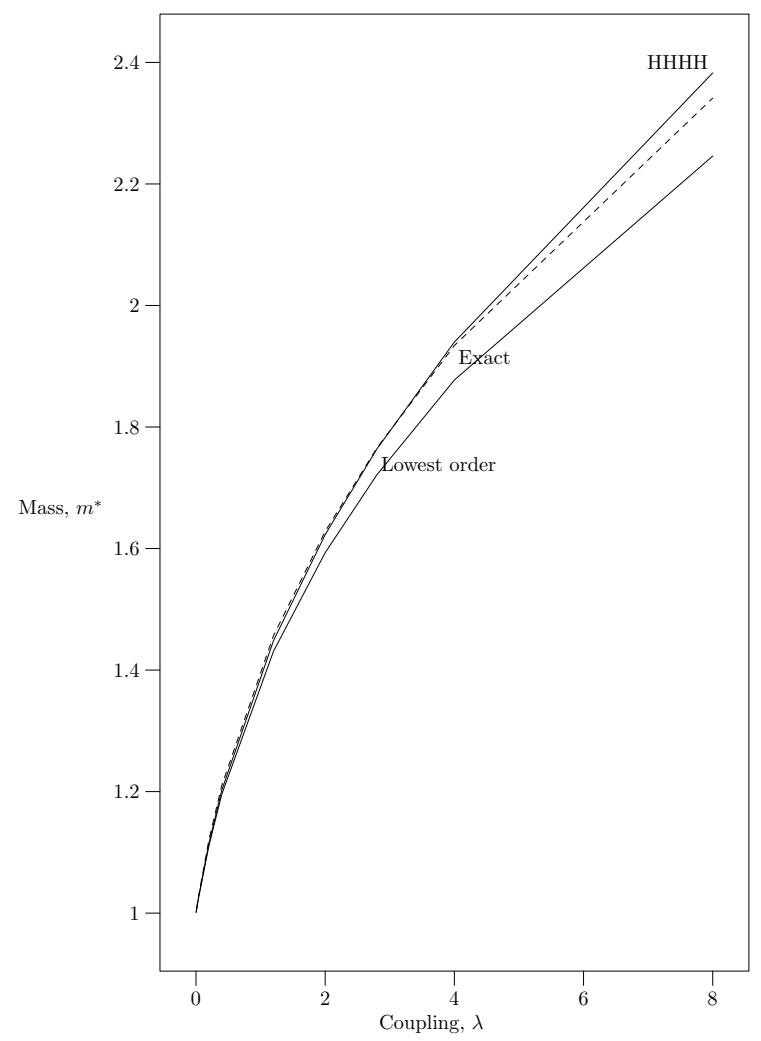

Figure 2. Comparison of improved ansätz with lowest order and exact answer for $\lambda \phi^{4}, d=1$

\section{CONCLUSIONS}

The Source Galerkin method promises to extend the range of field theoretic problems that can be solved with current computers. The low order results of the method applied to the nonlinear $\sigma$ model show asymptotic freedom and are close to the large- $N$ results. The contributions from higher order corrections are being studied. Understanding this model is of primary importance, as it is a step to supersymmetric and matrix models. One of the major advantages of our method lies in its approach to fermionic fields. Fermions are treated identically to bosons except for the anti-commutivity of the associated sources. Consequently, expansions involving fermions and bosons are symmetric in both types of fields and there is no analogue to the fermion determinant problem. The preliminary results of fermionic theories are very promising [7]. Source Galerkin takes advantage of the symmetries of a theory. The calculations are done in a continuum formulation, thus avoiding lattice-specific problems. While studying various ways of approximating Green's functions an interesting application has been investigated. Using Sinc-based spectral representations, we can calculate Feynman diagrams in a perturbation theory. Complicated diagrams can be evaluated much faster than with Monte-Carlo methods, and to higher accuracies 8].

\section{ACKNOWLEDGMENTS}

Computational work in support of this research was performed at the Theoretical Physics Computing Facility at Brown University and National Energy Research Scientific Computing Center. This work is supported by DOE contract DEFG0291ER40688, Tasks A and D.

\section{REFERENCES}

1. S. García, G. S. Guralnik, J. W. Lawson, Phys. Lett. B333 (1994), 119.

2. J. W. Lawson, G.S. Guralnik, Nuc. Phys. B459 (1996), 589. hep-th/9507130.

3. S. C. Hahn, G.S. Guralnik, hep-th/9804187.

4. S. C. Hahn, G.S. Guralnik, hep-th/9901019. 
5. C. A. J. Fletcher, Computational Galerkin Methods., Springer-Verlag.

6. F. Stenger, Numerical Methods Based on Sinc and Analytic Functions., Springer-Verlag.

7. J. W. Lawson, G.S. Guralnik, Nuc. Phys. B459 (1996), 612. hep-th/9507131.

8. R. Easther, G. Guralnik, S. Hahn, hep$\mathrm{ph} / 9903255$ (1999). 